\section{P132 HOSPITAL RE-ADMISSION IN PATIENTS WITH INFLAMMATORY BOWEL DISEASE - WHAT ARE THE RISK FACTORS?}

Renate Fromson, Rajan N Patel*, William Blad, Dariush Sadigh, Voishim Wong. The Whittington Hospital Nhs Trust, London, UK

\subsection{6/gutjnl-2020-bsgcampus.207}

Introduction Early re-admission after hospitalisation for an inflammatory bowel disease (IBD) flare is a negative quality indicator and causes unnecessary healthcare expense. Scoring systems to predict IBD readmissions have been shown to be ineffective. We aimed to describe the IBD re-admission rate at our hospital and investigate the risk factors.

Methods Retrospective study of patients admitted to a London-based district general hospital under the gastroenterology team with a flare of inflammatory bowel disease between 2015 and 2018. Characteristic including but not limited to demographics, disease type, length of stay (LOS) during index admission, biochemistry and biologic use were recorded. Hospital software (Sunquest Integrated Clinical Environment, Medway) was used to identify patients re-admitted at 30 and 90 days after discharge. Multivariate logistic regression was performed.

Results 138 patients were admitted with an IBD flare during the study period $(74(53.6 \%)$ Crohn's disease (CD), 56 $(40.6 \%)$ ulcerative colitis (UC), 8 (5.8\%) IBD-U). Median age 33.5 (IQR 26 - 52), 71 (51.4\%) female. Median LOS was 4.5 days (IQR 1.8 - 8). $36(26 \%)$ patients were taking a biologic. Re-admissions occurred within 30 days in 19 patients $(13.7 \%)$ and within 90 days in 30 patients (21.7\%). Multivariate logistic regression showed that a raised CRP on discharge was associated with re-admission. For every increased unit of CRP by one there was an increased risk of readmission by 1.1 times $(p=0.05)$. Patients aged $22-39$ were significantly less likely to be readmitted (OR: 0.38, $\mathrm{p}=0.015$ ). Male patients were significantly more likely to be readmitted (OR: 2.52, $\mathrm{p}=0.05)$.

Abstract P132 Table 1 Demographics and biochemistry associated with IBD re-admissions within 30 days of discharge.

\begin{tabular}{llll}
\hline & $\begin{array}{l}\text { 30-day Re-admission } \\
(\mathrm{n}=21)\end{array}$ & $\begin{array}{l}\text { No Re-admission } \\
(\mathrm{n}=117)\end{array}$ & $\mathrm{p}$ value \\
\hline Age, median (IQR) & $31(25.5-52)$ & $34(26-51)$ & 0.44 \\
Gender (F), n (\%) & $10(47.6)$ & $61(52.1)$ & 0.70 \\
Type of IBD (CD), n (\%) & $10(47.6)$ & $64(54.7)$ & 0.55 \\
LOS, days (median, IQR) & $3(0.5-11)$ & $5(2-8)$ & 0.35 \\
CRP on discharge (median, IQR) & $23(5.5-59.3)$ & $9(2-25)$ & 0.005 \\
Albumin on discharge & $37(31.5-41.5)$ & $36(31.3-41)$ & 0.45 \\
(median, IQR) & & & \\
Biologic use, n (\%) & $6(28.6)$ & $30(25.6)$ & 0.78 \\
\hline
\end{tabular}

Conclusion The 30 day and 90 day re-admission rate for our IBD population is just over $10 \%$ and $20 \%$, respectively. CRP at discharge is significantly associated with both 30 and 90 day re-admission. After adjusting for confounders; CRP, age older than 40 and male gender were associated with re-admission to hospital. We advise caution in discharging IBD patients with raised inflammatory markers. Close follow up within a few days of discharge would be appropriate in this high risk sub-group.

\section{P133 AN ATLAS OF CANONICAL CYTOKINE REGULATED TRANSCRIPTIONAL NETWORKS UNVEILS A NOVEL MOLECULAR STRATIFICATION OF IBD}

${ }^{1}$ Polychronis Pavlidis*, ${ }^{1}$ Anastasia Tsakmaki, ${ }^{2}$ Umar Niazi, ${ }^{2}$ Mansoor Saqi, ${ }^{1}$ Gavin Bewick, ${ }^{3}$ Nick Powell. ${ }^{1}$ King's Health Partners, London, UK; ${ }^{2}$ NIHR BRC Translational Bioinformatics, London, UK; ${ }^{3}$ Imperial College London, London, UK

\subsection{6/gutjpl-2020-bsgcampus.208}

Introduction The immune- epithelial interactions are central in our current understanding of inflammatory bowel disease (IBD) pathogenesis. Cytokines have been shown to play an integral part in this cross-talk, with some considered pro-inflammatory (e.g. IFN $\gamma, \mathrm{TNF} \alpha$ ) and others protective (e.g. IL22).

Methods We set out to define and compare the transcriptional programmes regulated by the canonical cytokines IFN $\gamma(\mathrm{TH} 1)$, IL13 (TH2), IL17A(TH17), IL22 (TH22) and TNFo, as a pro-inflammatory control, in human colonic organoids (colonoids) and associate them to disease phenotypes and therapeutic trajectories in IBD.

Whole transcriptome profiling of cytokine treated human colonoids $(n=4)$ was performed using the Illumina platform. Bioinformatic tools included: pathway analysis (Ingenuity database, interrogation of whole biopsy transcriptomic profiles of IBD patients $(n=423)$ using gene set variation analysis (GSVA) and weighted gene correlation network analysis (WGCNA).

Results A large functional overlap was found between IFN $\gamma$, IL22 and TNF $\alpha$ transcriptional programmes with key pathogenic pathways upregulated by all three cytokines (e.g. IL6, NF-kB, TREM1, TLR signalling, acute phase response, neutrophil chemotaxis). GSVA revealed enrichment of all cytokine regulated transcriptional modules in active inflammation while there was no difference in activated module number or type between UC and colonic CD. Intriguingly, patients with the same endoscopic activity had a gradient of activated cytokine regulated modules. Those with $\geq 2$ modules enriched had a higher risk of non-response to anti-TNF $\alpha$ therapy in both IBD phenotypes [relative risk: $2.9,95 \% \mathrm{CI}(1.7,6)]$. A strong, positive correlation was seen between IL22, IFN $\gamma$ and TNF $\alpha$ enrichment scores. WGCNA revealed a neutrophil chemotaxis chemokine module to be the pathway most strongly associated with anti-TNF $\alpha$ non-response. This module was primarily upregulated by both IL22 and TNF $\alpha$.

Conclusions Our study provides novel insights into the human gut immune-epithelial interactome and paves the way for a more granular immunophenotyping of IBD. It highlights that the simultaneous activation of modules regulated by multiple canonical cytokines is associated with non-response to antiTNF $\alpha$. Targeting of these shared pathogenic pathways may hold the key to overcoming non-response to biological therapies.

\section{P134 THE INFLAMMATORY BOWEL DISEASE BIORESOURCE: FOCUS ON RESEARCH FACILITATION}

1,2 Laetitia Pele*, ${ }^{1,2}$ Rachel Simpkins, ${ }^{1,2}$ Catherine Thorbinson, ${ }^{1,2}$ Deepthy Francis,

1,2Rasha Shawky, ${ }^{1,2}$ Miles Parkes, Genetics Consortium UK IBD. 'IBDD BioResource, NIHR BioResource, Cambridge, UK; ${ }^{2}$ Department of Medicine, University of Cambridge, UK

\subsection{6/gutjnl-2020-bsgcampus.209}

Introduction The Inflammatory Bowel Disease (IBD) BioResource is a UK-wide platform comprising $>32,000$ Crohn's 